\title{
CO-CREATING REQUIREMENTS AND ASSESSING END-USER ACCEPTABILITY OF A VOICE-BASED CHATBOT TO SUPPORT MENTAL HEALTH: A THEMATIC ANALYSIS OF A LIVING LAB WORKSHOP
}

\author{
PREPRINT
}

\begin{abstract}
Antonio Benítez-Guijarro, Raymond Bond, Frederick Booth, Zoraida Callejas, Edel Ennis, Anna Esposito, Matthias Kraus, Gavin McConvey, Michael McTear, Maurice Mulvenna, Courtney Potts, Louisa Pragst, Robin Turkington, Nicolas Wagner, Huiru Zheng
\end{abstract}

A. Benítez-Guijarro, Z. Callejas: University of Granada, Granada (Spain)

\{ajbenitez, zoraida\}@ugr.es

R. Bond, F. Booth, E. Ennis, M. McTear, M. Mulvenna, C. Potts,

R. Turkington, H. Zheng: Ulster University, Shore Road, Newtownabbey (Northern Ireland)

\{rb.bond, Booth-F, e.ennis, mf.mctear, md.mulvenna\}@ulster.ac.uk

\{c.pottsturkington-R, h.zheng\}@ulster.ac.uk

\section{A. Esposito}

Università degli Studi della Campania Luigi Vanvitelli, Caserta (Italy)

iiass.annaesp@tin.it

M. Kraus, L. Pragst, N. Wagner: University of Ulm, Ulm (Germany)

\{matthias.kraus, louisa.pragst, nicolas.wagner\}@uni-ulm.de

G. McConvey: Action Mental Health, Newtownards (Northern Ireland)

gmcconvey@amh.org.uk

This is a preprint of the paper:

Benítez-Guijarro A. et al. (2021) Co-creating Requirements and Assessing End-User Acceptability of a Voice-Based Chatbot to Support Mental Health: A Thematic Analysis of a Living Lab Workshop. In: D'Haro L.F., Callejas Z., Nakamura S. (eds) Conversational Dialogue Systems for the Next Decade. Lecture Notes in Electrical Engineering, vol 704. Springer, Singapore. https ://doi .org/10.1007/978-981-15-8395-7_15

This preprint follows Springer Self-archiving policy for non-open access books and chapters (https://www . springer com/gp/open-access/publication-policies/self-archiving-policy): "authors may deposit a portion of the pre-submission version of their manuscript (preprint) in a recognised preprint server (...). This portion of the pre-submission manuscript (preprint) may be deposited and made publicly available at any point." 


\begin{abstract}
Mental health and mental wellbeing have become an important factor to many citizens navigating their way through their environment and in the work place. New technology solutions such as chatbots are potential channels for supporting and coaching users to maintain a good state of mental wellbeing. Chatbots have the added value of providing social conversations and coaching 24/7 outside from conventional mental health services. However, little is known about the acceptability and user led requirements of this technology. This paper uses a living lab approach to elicit requirements, opinions and attitudes towards the use of chatbots for supporting mental health. The data collected was acquired from people living with anxiety or mild depression in a workshop setting. The audio of the workshop was recorded and a thematic analysis was carried out. The results are the co-created functional requirements and a number of use case scenarios that can be of interest to guide future development of chatbots in the mental health domain.
\end{abstract}

\title{
1 Introduction
}

Health should be considered in terms of mental wellbeing as well as the absence of mental disorders, with a healthcare focus on the understanding, promotion and protection of wellbeing [1]. With treatment, many people do experience recovery following an episode of mental illness. However, others may be described as "living with" mental ill health, and managing their own symptoms using coping strategies and their own support networks together with the formal support services and therapies such as counselling and medication. Thus, identification of relapse signs and symptoms of decline are important.

The use of digital health interventions, whether it is mobile or web-based applications, have the potential to improve access to mental healthcare, providing opportunities to engage and empower patients/users in treating mental ill health. As demand increases for mental health care, to the point where it exceeds available resources of national health care services, there is potential for digital health interventions to reduce waiting times to be seen by healthcare consultants/therapists through offering flexible and immediate means of accessing therapy or help [2].

One form of a digital intervention for mental ill health support and coaching are conversational systems, to which we will refer to as chatbot 11 . Chatbots can provide additional empowerment and support in the self-management of mental ill health by symptom checking at various times of the day and night, as well as rapid and appropriate interventions e.g. signposting to resources, appropriate feedback such as prompts, supportive messages, coping strategies and symptom summaries. Chatbots are also intuitive to interact with since they utilise natural language, which is the most natural form of human communication [3]. It is important to note that chatbots should not be considered full "virtual therapists", but have many advantages and can provide support to augment traditional services. In fact, current evidence shows there is a potential for conversational agents in psychiatric treatment [4].

There are a number of available chatbot systems which have been designed for monitoring of mental ill health symptoms, management of symptoms and guiding users through established therapy methods. For example, Woebot is a text-based chatbot technology which provides mood tracking and guides users through Cognitive Behavioural Therapy (CBT), provides exercises and stories to help facilitate improvement of users' mental wellbeing. Results showed that symptoms of depression and anxiety had reduced significantly over the course of the study period amongst Woebot users [5]. Another example of an existing text-based chatbot system is Wysa, which was co-designed by therapists, coaches, users and experts and comprises CBT, Dialetical Behavioural Therapy, mindfulness and various other behavioural reinforcement tools to help users build emotional resilience skills [6]. In addition, the study with the chatbot Vincent [7] found that caring for a chatbot (i.e. when the chatbot is the care receiver instead of the caregiver) enhances self-compassion.

While most existing chatbot technologies for mental health purposes operate on a text basis [8], there is interest in developing chatbot technology for mental health that incorporates the use of audio analysis which can infer mood, sentiment, affect or mental illness from speech data. There are particular verbal and non-verbal (prosodic/ suprasegmental) speech features which are considered robust markers for depressive states and can allow learning algorithms to discriminate between depressed and healthy people using speech [9]. Based on current evidence, automated classification of speech could be used as an objective measure of depression and other mental illnesses and therefore help improve diagnosis accuracy.

The work described in this paper is part of the H2020 MSCA RISE project MENHIR: Mental health monitoring through interactive conversations (ref. no. 823907, https://menhir-project.eu). MENHIR consists of a consortium of

\footnotetext{
${ }^{1}$ There are slight differences between these terms, specially in their academic use as explained in [3], but we use the term 'chatbot' because it is more familiar to the end user and thus facilitates co-creation approaches.
} 
eight partners, which includes five academic institutions, two companies and a non-profit organisation. The technology that will be studied and developed in MENHIR is designed together with persons with mental ill health, experts and caregivers. The underlying principle is that nobody knows better about their needs and preferences than users, which is why they play an influential role in the MENHIR project through co-creation activities.

Co-creation refers to involving stakeholders throughout the design. This can include a series of home visits, focus groups, workshops, in-depth interviews, cultural probes and technology biographies. Co-creation provides valuable information for understanding the user needs and collecting requirements. The aim of this paper is to assess the acceptability and co-create requirements for a voice-based chatbot. We have used thematic analysis of data collected at a user-centred workshop involving clients of Action Mental Health, an organisation in Northern Ireland which assists people with mental ill health.

\section{Methods}

\subsection{Study protocol}

As indicated in [10], living labs are "a collection of people, equipment, services and technology to provide a test platform for research and experiments" that may be employed for a number of purposes including sharing ideas, engagement with user communities and other stakeholders, co-creation, data collection and evaluation.

Co-creation can be defined as the collaborative knowledge generation by academics working alongside other stakeholders [11]. As indicated in [12], co-creation allows creating partnerships between researchers and the people for whom the research is ultimately meant to be of use, in our case people with anxiety or mild depression. The authors underline that not using this type of participatory approaches delays the implementation of effective practices, in this case in mental healthcare, which "affects people's health and contributes to the unsustainability of the health system".

This paper outlines the outcomes of a co-creation workshop using the living-lab methodology which involved the potential users of the chatbot. This piece of research involved a workshop to elicit opinions and co-create requirements for a speech-based mental health chatbot service. The workshop was carried out with Action Mental Health (AMH) clients from New Horizons Newtownards on the 27th June 2019.

AMH is an organisation that assists people with mental ill health in order to offer them services and mechanisms that allow them to manage their conditions. AMH has several centers spread across Northern Ireland, each of them offering different activities and services. AMH clients are often referred from clinical mental health centers with different reports and personal information (reports about their diagnosis, mental and personal clinical history, risk factors, traumas, critical background and other sensitive data).

Ethical approval for this workshop was granted by Ulster University and ethics is managed by MENHIR's Ethics Board. The workshop session had a duration of approximately 2 hours. The participants included 9 clients from AMH New Horizon Newtownards, a key worker from AMH New Horizon Newtownards and 3 researchers from the MENHIR project who presented the project and acted as facilitators. All 9 clients gave their consent for the group discussion to be recorded.

From the different models of co-creation described in [11], we used two: experience based co-design and technology co-design, as the goal of the sessions was two-fold: to use the clients' experience as the starting point to design the role of the chatbot, and to develop a technology that is acceptable, fits a purpose and is tailored to its users' capabilities and what matters to them.

The workshop had the following structure:

1. Welcome and brief introduction to the project.

2. Demonstration of a mental health chatbot and smart speaker by workshop facilitator.

3. Completion of consent form and study proforma.

4. Group discussion:

- Strengths and limitations of this type of chatbot.

- Who would use this chatbot?

- Which type of people would the chatbot suit?

- Could it be used in conjunction with AMH mental health support services? (how?)

- What are the pros and cons of using chatbot in the context of mental ill health?

- What features should a mental health chatbot have? 
- What kind of conversations would you want to have with a chatbot?

- What kind of persona/s should the chatbot have?

To facilitate the interaction, at the beginning of the group discussion, the participants were given green and pink post-it notes where they could write the benefits (green) and limitations (pink) of the technology. The audio of the group discussion was recorded and then interpreted using thematic analysis.

\section{Context of Action Mental Health (AMH)}

AMH clients are usually at a medium-low risk level. These clients attend a local AMH center and are interviewed. During this interview the workers of the center explain the types of services offered in the center and ask the clients about their hobbies, goals, schedules and other personal information to understand the activities and therapies that may be of interest.

With this information, they generate an action plan to which the client commits. With the action plan, the user identifies their long term goals and set dates to achieve them. The means for achieving the objectives is to engage in vocation and personal development activities.

Once the action plan is established, the centers will offer clients up to two years of assistance. During this period, patients attend the AMH center several times per week to carry out the activities planned (e.g. gardening, handcrafting, computer science, cooking, etc.).

Although the action plan is a concept developed by $\mathrm{AMH}$, it could be easily used in other settings and can be of great interest not only for the chatbot technology developed in MENHIR, but for general use, as it provides a means to ground motivational feedback in the user objectives and hobbies and structured means of achieving them by means of scheduled activities.

\section{Results of the thematic analysis}

There were 4 main topics of discussion within the workshop, 1) challenges faced by people with ill mental health, 2) chatbot functionalities, 3) chatbot characteristics, and 4) use of the chatbot.

The strengths and limitations of chatbots for mental health as reported by the participants in the post-it notes are presented in Table 1, where the statements corresponding to the same person are marked with the same number.

The group discussion was recorded resulting in a 1 hour and 18 minute audio recording that was then analyzed using thematic analysis. Thematic analysis is one of the most commonly used methods of analysis in qualitative research and focuses on identifying themes within transcribed data, usually resulting from interviews and focus groups [13]. The topics and themes identified have been listed in Table 2 In total, there were 17 themes identified from the topics of the group discussion.

\section{Functional requirements}

Based on the thematic analysis and the literature, a number of requirements have been discovered to deliver chatbot mental health technologies. These requirements have been outlined as follows.

Chatbots should have mechanisms for allowing to collect different types of data, with the user's permission. Permissions such as recording of the user's voice, collecting user demographics, information on their daily habits and experiences. Chatbots should also allow users to decide if the system can interpret their current state and if the system should gather information about emergency contacts and whether to permit the system to save the data to train a user model to be used for posterior interactions. Chatbots should also provide mechanisms that allow users to decide what information can be shared with their key workers (i.e. counsellors, psychiatrists) and under which conditions. Moreover, they should be able to start a conversation with the user proactively according to an event, programmed frequency or routine. Also they should be able to start/resume a conversation upon the user request, or set to a sleep mode upon user request. The frequency of engagement with the chatbot should be customisable.

An additional requirement is that the chatbot should provide the users a space where they can express their thoughts and feelings, be able to assess the emotional state and track the changes in the user's emotional state over time. As well, the system should be able to detect potential risks within the user's interactions and during the mood tracking.

The chatbot should be aware of the users action plans and preferences, as set by the users and/or their key workers/counsellors. In conjunction with this, the system should remind and motivate the user to engage in all aspects of 
Table 1: Result of the strengths vs. limitations activity reproduced verbatim

\begin{tabular}{|c|c|}
\hline Strengths & Limitations \\
\hline 1. Akin to "talking to the dog" & 1. Ability to be exploited \\
\hline 1. Anonymous and secure & $\begin{array}{l}\text { 1. Flaws where chatbots have sent recordings } \\
\text { to contacts }\end{array}$ \\
\hline \multicolumn{2}{|l|}{ 1. Avoidance of stigma } \\
\hline \multicolumn{2}{|l|}{$\begin{array}{l}\text { 1. Potential to recognize poor men- } \\
\text { tal health when the individual can't }\end{array}$} \\
\hline \multicolumn{2}{|l|}{ 1. Voice recognition for security } \\
\hline \multicolumn{2}{|l|}{$\begin{array}{l}\text { 1. Potential for voice modulation } \\
\text { detection (distress, anxious, etc.) }\end{array}$} \\
\hline \multicolumn{2}{|l|}{$\begin{array}{l}\text { 1. Ability to know when to "inter- } \\
\text { vene" (e.g. emergency) }\end{array}$} \\
\hline $\begin{array}{l}\text { 2. As a companion when there is } \\
\text { nobody to talk to }\end{array}$ & $\begin{array}{l}\text { 2. Individual privacy and wider cryptographic } \\
\text { protection }\end{array}$ \\
\hline $\begin{array}{l}\text { 2. Potentially provide an answer to } \\
\text { a situation }\end{array}$ & 2. An establishment of absolute trust \\
\hline $\begin{array}{l}\text { 3. Trusting Alexa making sure you } \\
\text { are talking to people with proper } \\
\text { identification }\end{array}$ & 3. Conversation cannot flow \\
\hline & 3. Making sure you have privacy \\
\hline
\end{tabular}

4. Someone/thing to talk to in confidence. It is easier at times to talk to someone/thing anonymously. Don't like to off load to family and friends. They don't always understand

\begin{tabular}{|c|c|}
\hline 5. Support on hand & 5. Robotic voice \\
\hline 5. Unbiased & 5. Privacy \\
\hline $\begin{array}{l}\text { 6. Can provide reminders to take } \\
\text { medication, attend appointments } \\
\text { or engage in helpful activities e.g. } \\
\text { "have your remembered to medicate } \\
\text { today?" }\end{array}$ & $\begin{array}{l}\text { 6. The user receives responses without being } \\
\text { sure where their responses are being taken from, } \\
\text { are they from a reliable source? }\end{array}$ \\
\hline $\begin{array}{l}\text { 7. Takes at the necessity of relying } \\
\text { on flawed human memory the chat- } \\
\text { bot could give automatic reminders } \\
\text { for things like doctor's appointments } \\
\text { and the need to take medication and } \\
\text { even things as simple as a reminder } \\
\text { for some self-care e.g. to eat a meal } \\
\text { or take a shower }\end{array}$ & $\begin{array}{l}\text { 7. A chatbot cannot be the same as genuine one } \\
\text { to one human contact. It is unrealistic to expect } \\
\text { technology to be able to understand the subtle } \\
\text { nuances of emotion, feelings or the intricacies } \\
\text { of speech }\end{array}$ \\
\hline $\begin{array}{l}\text { 8. Support and understanding from } \\
\text { people that are or have been in the } \\
\text { same situation }\end{array}$ & $\begin{array}{l}\text { 8. Can it run } 24 / 7 ? \text { and if we come across an } \\
\text { issue can we report it? }\end{array}$ \\
\hline $\begin{array}{l}\text { 9. Helping someone in need of con- } \\
\text { versation or advice if nothing or no- } \\
\text { one is available. }\end{array}$ & $\begin{array}{l}\text { 9. Would it report to authorities to prevent } \\
\text { suicide or self harm - or harm to others }\end{array}$ \\
\hline $\begin{array}{l}\text { 9. Some people don't like to share } \\
\text { their emotions in a group }\end{array}$ & \\
\hline
\end{tabular}


Table 2: Topics and themes identified from the workshop

\begin{tabular}{ll}
\hline Topics & Themes \\
Challenges faced by people with & 1. Isolation \\
mental ill health & 2. Difficulty for honest disclosure \\
\hline Chatbot functionalities & 3. Symptom recognition \\
& 4. Continuous monitoring \\
& 5. Disclosure facilitation \\
& 6. Companionship/active listening \\
7. Risk detection
\end{tabular}

their action plan. The system must have reminding and motivational functionalities which are bespoke to the users needs. The system should be able to remind and explain to the user of the benefits of adhering to the plan, while also suggesting new activities to engage in.

\section{Discussion}

Based on the requirements elicitation and thematic analysis, we developed a number of potential use case scenarios for an envisioned mental health chatbot system. We describe the two main ones.

\subsection{Use case scenario 1: Intelligent Reminders}

Users have stated that motivation to keep their daily tasks is key for their recovery. Therefore, a kind of companion is wished for, that is used as a daily planner for activities and tasks and has a motivation and a reminder functionality. Furthermore, these functionalities should be personalised in order to be fully accepted. In MENHIR, we will develop a sophisticated reminder for actively encouraging users to engage in activities related to their action plan. As it may not always be perceived positive to remind, for some users it may be better to let them have the responsibility of remembering their appointments. Therefore, a strategy that considers the type of user (e.g. personality, mood, activity history) will be considered. Among the reminder options, we will investigate explicit reminders that address different motivational aspects (e.g. pragmatic/emotional explanations, why engaging in activities is beneficial or necessary), and we will also explore the possibility to include implicit approaches to stimulate the memory of the user by indirect cues so that users are responsible for remembering their schedules.

A major challenge of our approach will be to find strategies that establish trust between the human user and the chatbot. Otherwise, reminders will be perceived as obstructive or obtrusive and the MENHIR chatbot as a whole will not be accepted. In order to fulfil this requirement, it is essential to develop appropriate timing strategies. Timing will be based on an event, a programmed frequency or a learned routine. Furthermore, the reminder functionality will be configurable and users should be able to put the chatbot in sleep mode. For motivating users, the dialogue systems needs access to individual action plans and preferences. Additionally, tracked user emotion could be used to adapt the motivational strategies.

For illustrating a strategy that considers user-dependent features as well as different kinds of motivation, we provide the following example: 
According to her action plan, Anna is to attend to glass painting classes every Wednesday at $10.00 \mathrm{am}$. To arrive on time, Anna must leave her house at $9.00 \mathrm{am}$. In this use case, the system typically reminds her at $8.00 \mathrm{am}$ according to a predefined frequency. Depending on the type of users different types of reminding are foreseeable:

Option A (Pragmatic Reminder): MENHIR: Hi Anna, remember you have to leave in an hour for your glass class. Today you will learn how to colour your piece.

Option B (Emotional Reminder): MENHIR: Hi Anna, remember you have to leave in an hour for your glass class. You will have a very good time because today you will be colouring your piece. This will be the last step to achieve your objective to make your own lamp. You can be proud of yourself.

Option C (Indirect Reminder): MENHIR: Hi Anna, do you have any activities programmed for today?

Option D: - Not to remind -

\subsection{Use case scenario 2: user diary}

The analysis of the co-creation workshop shows that users would value a chatbot that offers them companionship. They want to talk about their struggles without being judged or feeling that they are a burden to others. A chatbot can facilitate the disclosure of their thoughts, as it is inanimate and therefore impartial and permanently available without becoming fatigued.

In MENHIR, we will consider a diary-like functionality that allows users to talk about their day while displaying active listening behaviour as a way to address those needs. The main objective of this use case is to get the user talking about their day by open-ended and engaging questions, as well as to keep them talking by appropriate backchanneling and follow-up questions so that enough data is obtained to perform the automatic analysis of their state and progress. If the diary is used on a regular basis, it can be used to attend to the users' need for monitoring their mental state.

An account of daily occurrences is likely to offer sufficient material to perform emotional recognition using linguistic and paralinguistic information as mentioned before. We can also enrich the data obtained with other resources available for the community, such as those of the Audio/Visual Emotion Challenge and Workshop, which has a sub-challenge devoted to detecting depressive states [14].

A thorough understanding of the diary entry of the user is thus not within the scope of MENHIR, and was in fact not a requisite for users. Therefore, the main challenge of this use case is finding appropriate questions that signal engagement without responding to the specific semantic content conveyed by the user.

A dialogue in this use case will typically start with the chatbot proactively approaching the user to ask about their day. The question must be chosen in a way that builds rapport and does not expect a positive or negative answer, so that the user feels comfortable to talk about whatever is on their mind. There must be also variety in the questions used for opening the dialogue, to foster long-term interactions. If the user talks for a longer period of time, appropriate backchanneling shows engagement and supports building a companionship between user and system. For short answers, the system needs to ask follow-up questions that favour a more extensive answer. As a semantic analysis of every possible user utterance is not within the scope of MENHIR, the follow-up questions must be generally applicable and can at most rely on an emotional analysis if the utterance allows for it.

An example dialogue of such a scenario could be:

MENHIR: Hi Anna, how was your day?

Anna: Not so good.

MENHIR: Did something happen?

Anna: Yesterday I had an argument with my mother and I have been feeling bad all day...

MENHIR: I'm sorry to hear that. You can tell me about it if that makes you feel better.

Anna: ... 


\section{Conclusion}

In this paper we have presented the results of a co-creation workshop in which scientists and people with mild anxiety and depression have collaborated to understand the acceptability and requirements for the development of mental health chatbots.

Results from the workshop were analysed using thematic analysis to highlight the key themes of discussion, from which we have obtained a list of 17 themes related to challenges that could be addressed (isolation and honest disclosure), functionalities (symptom recognition and monitoring, companionship, risk detection), characteristics (including personalization, proactiveness, accessibility, and privacy) and usage conditions (cost, access, intention to use). Our results provide interesting insights for the development of a mental health chatbot, including requirements and use case scenarios, from which we have highlighted and described the intelligent reminder and user diary use cases.

For future work, we will develop the dialogues in the scenarios presented. The first challenges that we will address will be the development of personalized dialogue management strategies based on representations of the user actions plans for the intelligent reminder scenario as well as the multimodal analysis of interaction recordings in the user diary scenario. Once the scenarios are designed, they will be validated with key workers and assessed in further co-creation workshops and Wizard-of-Oz evaluation sessions. For these sessions we will consider, among others, the aspects of informational support, emotional support, positive support, skill building and potential negative experiences studied recently in [15].

\section{Acknowledgements}

This research has received funding from the European Union's Horizon 2020 research and innovation programme under grant agreement No 823907 (MENHIR: Mental health monitoring through interactive conversations https: //menhir-project.eu).

\section{References}

[1] World Health Organization, Victorian Health Promotion Foundation, and University of Melbourne. Promoting mental health: concepts, emerging evidence and practice. World Health Organization, 2004.

[2] Chris Hollis, Richard Morriss, Jennifer Martin, Sarah Amani, Rebecca Cotton, Mike Denis, and ShÃŽn Lewis. Technological innovations in mental healthcare: harnessing the digital revolution. The British Journal of Psychiatry: The Journal of Mental Science, 206(4):263-265, April 2015.

[3] Michael McTear, Zoraida Callejas, and David Griol Barres. The Conversational Interface: Talking to Smart Devices. Springer International Publishing, 2016.

[4] Aditya Nrusimha Vaidyam, Hannah Wisniewski, John David Halamka, Matcheri S. Kashavan, and John Blake Torous. Chatbots and Conversational Agents in Mental Health: A Review of the Psychiatric Landscape. The Canadian Journal of Psychiatry, 64(7):456-464, 2019.

[5] Kathleen Kara Fitzpatrick, Alison Darcy, and Molly Vierhile. Delivering Cognitive Behavior Therapy to Young Adults With Symptoms of Depression and Anxiety Using a Fully Automated Conversational Agent (Woebot): A Randomized Controlled Trial. JMIR mental health, 4(2), 2017.

[6] Becky Inkster, Shubhankar Sarda, and Vinod Subramanian. An Empathy-Driven, Conversational Artificial Intelligence Agent (Wysa) for Digital Mental Well-Being: Real-World Data Evaluation Mixed-Methods Study. JMIR mHealth and uHealth, 6(11):e12106, 2018.

[7] Minha Lee, Sander Ackermans, Nena van As, Hanwen Chang, Enzo Lucas, and Wijnand IJsselsteijn. Caring for Vincent: A Chatbot for Self-Compassion. In Proceedings of the 2019 CHI Conference on Human Factors in Computing Systems, CHI '19, pages 1-13, Glasgow, Scotland Uk, 2019.

[8] Alaa A. Abd-alrazaq, Mohannad Alajlani, Ali Abdallah Alalwan, Bridgette M. Bewick, Peter Gardner, and Mowafa Househ. An overview of the features of chatbots in mental health: A scoping review. International Journal of Medical Informatics, 132:103978, 2019.

[9] Nicholas Cummins, Stefan Scherer, Jarek Krajewski, Sebastian Schnieder, Julien Epps, and Thomas F. Quatieri. A review of depression and suicide risk assessment using speech analysis. Speech Communication, 71:10-49, 2015.

[10] Maurice D. Mulvenna, Birgitta Bergvall-Kåreborn, Brendan Galbraith, Jonathan Wallace, and Suzanne Martin. Living labs are innovation catalysts. In Robert J. Howlett, editor, Innovation through Knowledge Transfer 2010, pages 253-264, Berlin, Heidelberg, 2011. Springer Berlin Heidelberg. 
[11] Trisha Greenhalgh, Claire Jackson, Sara Shaw, and Tina Janamian. Achieving Research Impact Through Cocreation in Community-Based Health Services: Literature Review and Case Study. The Milbank Quarterly, 94(2):392-429, 2016.

[12] Janet Jull, Audrey Giles, and Ian D. Graham. Community-based participatory research and integrated knowledge translation: advancing the co-creation of knowledge. Implementation Science, 12(1):150, December 2017.

[13] Greg Guest, Kathleen M. MacQueen, and Emily E. Namey. Applied Thematic Analysis. SAGE Publications, 2011.

[14] Fabien Ringeval, Björn Schuller, Michel Valstar, Nicholas Cummins, Roddy Cowie, Leili Tavabi, Maximilian Schmitt, , Sina Alisamir, Shahin Amiriparian, Eva-Maria Messner, Siyang Song, Shuo Liu, Ziping Zhao, Adria Mallol-Ragolta, Zhao Ren, Mohammad Soleymani, and Maja Pantic. AVEC 2019 Workshop and Challenge: State-of-Mind, Detecting Depression with AI, and Cross-Cultural Affect Recognition. In Proceedings of AVEC'19, Nice, France, 2019. ACM Press.

[15] Vivian Ta, Caroline Griffith, Carolynn Boatfield, Xinyu Wang, Maria Civitello, Haley Bader, Esther DeCero, and Alexia Loggarakis. User experiences of social support from companion chatbots in everyday contexts: Thematic analysis. Journal of Medical Internet Research, 22(3), Mar 2020. 